\title{
La ciencia de cara a la integralidad en la asistencia
}

Este año, el $35^{\circ}$ Congreso Uruguayo de Cardiología se realizará del 7 al 9 de noviembre en el Convention \& Exhibition Center de Punta del Este.

Desde el Comité Organizador hemos pensado en un congreso con perfil plural e integrador con otras especialidades médicas, tratando de focalizar en temas de interés para la mayoría de los cardiólogos.

El mensaje que queremos transmitir es el concepto de la integralidad en la asistencia del paciente. Importante definición en el sistema sanitario que abarca las diferentes instancias asistenciales, desde la atención primaria a los procedimientos altamente especializados.

En este contexto, desarrollaremos temas como Prevención primaria, Insuficiencia cardíaca, Cardio-oncología, Cardiopatía isquémica-intervencionismo, Cirugía cardíaca y Cuidados posoperatorios, así como la Enfermedad cardiovascular en la mujer.

El curso precongreso se realizará el miércoles 6 de noviembre y, como es habitual, será la última actividad anual de la Comisión de Educación Médica Continua. En esta oportunidad el tema propuesto es Arritmias en la emergencia, un tema actual y siempre desafiante.

Los participantes tendrán la oportunidad de contar en sus dispositivos móviles con la App del congreso y una plataforma web con toda la información que se actualizará de forma periódica, simultáneamente con los avances del comité organizador.

Como novedad, tendremos la posibilidad de transmitir por teleconferencia las principales actividades del congreso, lo que permitirá el acceso remoto a las mismas.

El Congreso de la Sociedad Uruguaya de Cardiología, como es tradicional, brinda a cardiólogos, cirujanos cardíacos y médicos en general la oportunidad de actualizar sus conocimientos; hemos elaborado un programa muy rico y variado en cuanto a la temática científica, que a su vez contempla el relacionamiento de nuestra Sociedad con organizaciones cardiológicas internacionales.

Se llevarán a cabo simposios conjuntos de primordial importancia con la Sociedad Europea de Cardiología (ESC), con la Sociedad Española de Cardiología (SEC), con el Colegio Americano de Cardiología (ACC), con la Asociación Americana de Cirugía Torácica (AATS) y con la Clínica Mayo.

Contaremos con invitados de excepcional jerarquía procedentes del Cono Sur de nuestro continente, de Estados Unidos y de Europa, entre ellos, los Dres. Gorav Ailawadi, Abdallah El Sabbagh, Luis Allen, Daniel Philbin (Estados Unidos), Lina Badimón y Ángel Cequier Fillat (España), Jeroen Bax (Holanda) y Daniela Cardinale (Italia), que expondrán sobre temas de actualidad y compartirán mesas con destacados cardiólogos y cirujanos cardíacos locales.

Como en años anteriores, se incorporarán simposios de centros de medicina altamente especializada con propuestas de actualidad y del quehacer de cada centro, exponiendo la realidad de la cardiología y la cirugía cardíaca de nuestro país.

También desarrollaremos una actividad con el Centro Cardiovascular Universitario (Universidad de la República) y con los diferentes servicios de cardiología de la Administración de los Servicios de Salud del Estado de forma tal que conozcamos las actividades desarrolladas en estos centros que cubren la asistencia de gran parte de la población nacional.

Para esta amplia propuesta -la más importante del año en cardiología- contamos con su participación, de manera que el congreso sea el mejor ámbito de reunión y de innovación de conocimiento en toda su expresión.

Los saludamos afectuosamente. 